\title{
Random Forest Classifier for Extracting Water bodies from Pansharpened Image to Detect Surface Water Changes
}

\author{
K. Kalaivani, Asnath Victy Phamila, Sathish Kumar Selvaperumal
}

\begin{abstract}
Change detection from time series multispectral Landsat imagery has been an active research in remote sensing for several years to monitor the ecosystem, environment, climate and so on. This study is focused on detecting the changes in surface water by the integration of fusion and image classification techniques in multi-temporal multispectral Landsat images. The panchromatic band and the multispectral band of Landsat OLI and TM images respectively, were fused using undecimated wavelet transform to get the pan-sharpened image. Then classification techniques like Maximum Likelihood, Support Vector Machine, Artificial Neural Network and Random Forest were employed for extracting the water pixels and changed pixels. The performances of these classification techniques were analyzed based on metrics such as overall error, commission error, precision, recall, overall accuracy, kappa coefficients and the results show that the application of random forest classifier on pansharpened image outperforms in extracting the water pixels and also in highlighting the changes with maximum accuracy.
\end{abstract}

Keywords: Change detection, image classification, pansharpening, random forest, surface water.

\section{INTRODUCTION}

Frequent monitoring of surface water and reliable information is essential for various application like hydrology, Land Use Land Cover Change, assessment of water resources, wet-land mapping, water survey and management, environment monitoring and so on [1]. In recent decades, extracting surface water and detecting changes were possible with the help of satellite remote sensing at different spatial and spectral resolutions. Various image-processing techniques were available to extract surface water features from satellite images [2].

In this paper, pansharpened image is classified for identifying changes in surface water from multi-temporal satellite data.

Revised Manuscript Received on October 30, 2019

* Correspondence Author

K.Kalaivani*, Research Scholar, School of Computing Science and Engineering, VIT University, Chennai, India

Assistant Professor, Department of Computer Science and Engineering, Vels Institute of Science, Technology and Advance Studies, Chennai, India, Email: kalaivani_k@outlook.com

Asnath Victy Phamila, Associate Professor, School of Computing Science and Engineering, VIT University, Chennai, India. Email: asnathvicty.phamila@vit.ac.in

Sathish Kumar Selvaperumal, Associate Professor, Asia Pacific University Technology Park Malaysia, Kuala Lumpur, Malaysia. Email: sathish@staffemail.apu.edu.my

(c) The Authors. Published by Blue Eyes Intelligence Engineering and Sciences Publication (BEIESP). This is an open access article under the CC BY-NC-ND license (http://creativecommons.org/licenses/by-nc-nd/4.0/)
Pixel level image fusion is the process of combining different images of the same scene based on pixel-by-pixel to provide a single fused image more informative than any of the input images [3]. Pixel level image fusion is also termed as Pansharpening [4].

Pansharpening techniques are employed to sharpen low spatial resolution multispectral image with high spatial resolution panchromatic image to form a more detailed image more suitable for change detection. Existing satellite remote sensors, the Operational Land Imager (OLI), Enhanced Thematic Mapper Plus (ETM+) and Thematic Mapper (TM) sensors by the Landsat 8, 7 and 5 satellites, respectively, are extensively used for assessing the water quality assessment [5]. The data acquired by Landsat 5 and 8 were utilized for this study. The details of the wavelength range and spatial resolution of Landsat 5 and 8 bands are shown in Table 1 and 2 respectively.

Landsat 5 TM consists of seven spectral bands with a spatial resolution of $30 \mathrm{~m}$ for the bands $1-5$ and 7 . The thermal infrared band 6 has a spatial resolution of $120 \mathrm{~m}$ and it is resampled to $30 \mathrm{~m}$ (Table 1). Landsat $8 \mathrm{OLI}$ consists of nine spectral bands with a spatial resolution of $30 \mathrm{~m}$ for the bands 1-7 and 9. The thermal infrared band 10 and 11 has a spatial resolution of $100 \mathrm{~m}$. The panchromatic band 8 has a spatial resolution of $15 \mathrm{~m}$ (Table 2).

To assess the effectiveness of the proposed methodology, the geographical location of the saline Lake Urmia located in northwest of Iran is considered. Three scenes of Lake Urmia corresponding to the path/row - 168/34, 169/33 and 169/34 of the years 2010 and 2018 acquired in August 2010 and September 2018 were collected from USGS Global Visualization Viewer (https://glovis.usgs.gov). The multispectral images of Lake Urmia in 2000 and 2018 are shown in Fig. 1a and b.

\section{METHODOLOGY}

The proposed methodology involves three phases: i. preprocessing, ii. pansharpening, iii. image classification and it is implemented using MATLAB, $\mathrm{R}$ and QGIS. The workflow and the methods adopted are depicted in Fig.2. While preprocessing, the steps such as radiometric calibration, atmospheric correction, dark object subtraction, mosaicking, co-registration, resampling and clipping were performed [6]. The multispectral band of $30 \mathrm{~m}$ resolution and panchromatic band of $15 \mathrm{~m}$ resolution images were preprocessed and converted from at-satellite radiance to atmospheric reflectance and then to surface reflectance using dark object subtraction method. 
Random Forest Classifier for Extracting Water bodies from Pansharpened Image to Detect Surface Water Changes

Table- 1: Wavelength Range and Spatial Resolution of Landsat 5

\begin{tabular}{ccc}
\hline Band & Wavelength (Micrometers) & Resolution (Meters) \\
\hline Band 1 - Blue & $0.45-0.52$ & 30 \\
Band 2 - Green & $0.52-0.60$ & 30 \\
Band 3 - Red & $0.63-0.69$ & 30 \\
Band 4 - Near Infrared & $0.76-0.90$ & 30 \\
Band 5 - Shortwave Infrared 1 & $1.55-1.75$ & 30 \\
Band 6 - Thermal Infrared & $10.40-12.50$ & 120 (30) \\
Band 7 - Shortwave Infrared 2 & $2.08-2.35$ & 30 \\
\hline Table 2 - Wavelength Range and Spatial Resolution of Landsat 8 \\
\hline Band & Wavelength (Micrometers) & Resolution (meters) \\
\hline Band 1 - Coastal Aerosol & $0.43-0.52$ & 30 \\
Band 2 - Blue & $0.45-0.51$ & 30 \\
Band 3 - Green & $0.53-0.59$ & 30 \\
Band 4 - Red & $0.64-0.67$ & 30 \\
Band 5 - Near Infrared & $0.85-0.88$ & 30 \\
Band 6 - ShortWave Infrared 1 & $1.57-1.65$ & 30 \\
Band 7 - ShortWave Infrared 2 & $2.11-2.29$ & 30 \\
Band 8 - Panchromatic & $0.50-0.68$ & 15 \\
Band 9 - Cirrus & $1.36-1.38$ & 30 \\
Band 10 - Thermal Infrared 1 & $10.6-11.19$ & 100 \\
Band 11 - Thermal Infrared 2 & $11.50-12.51$ & 100 \\
\hline & & 30 \\
\hline
\end{tabular}

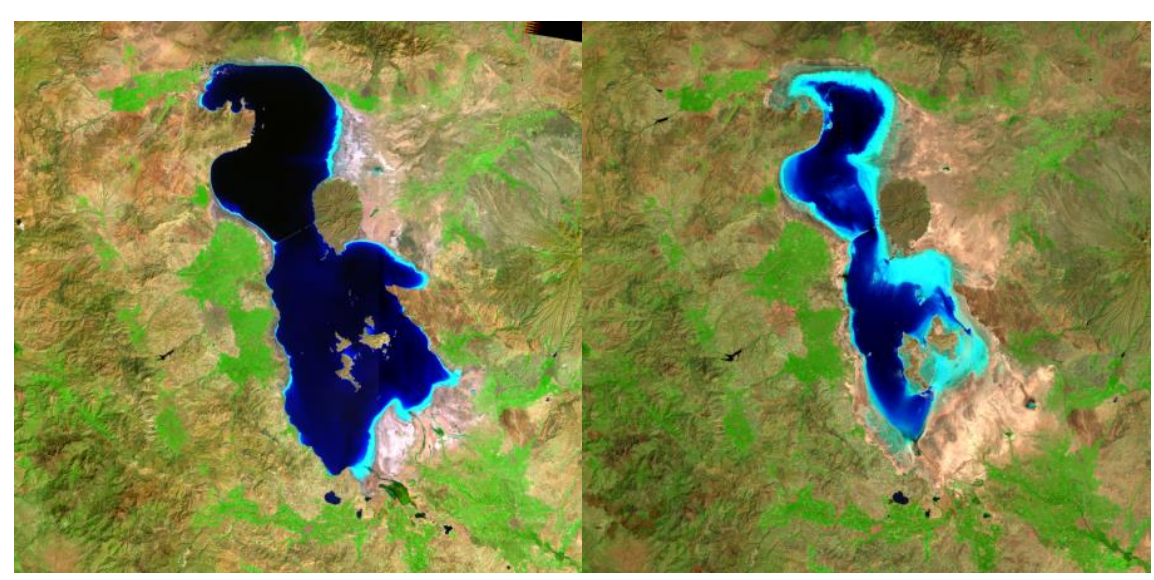

(a)

(b)

Fig. 1: (a) Lake Urmia in 2010, (b) Lake Urmia in 2018 
RADIOMETRIC CALIBRATION \&

ATMOSPHERIC CORRECTION

At Satellite Radiance to Top of Atmospheric Reflectance and Brightness Temperature

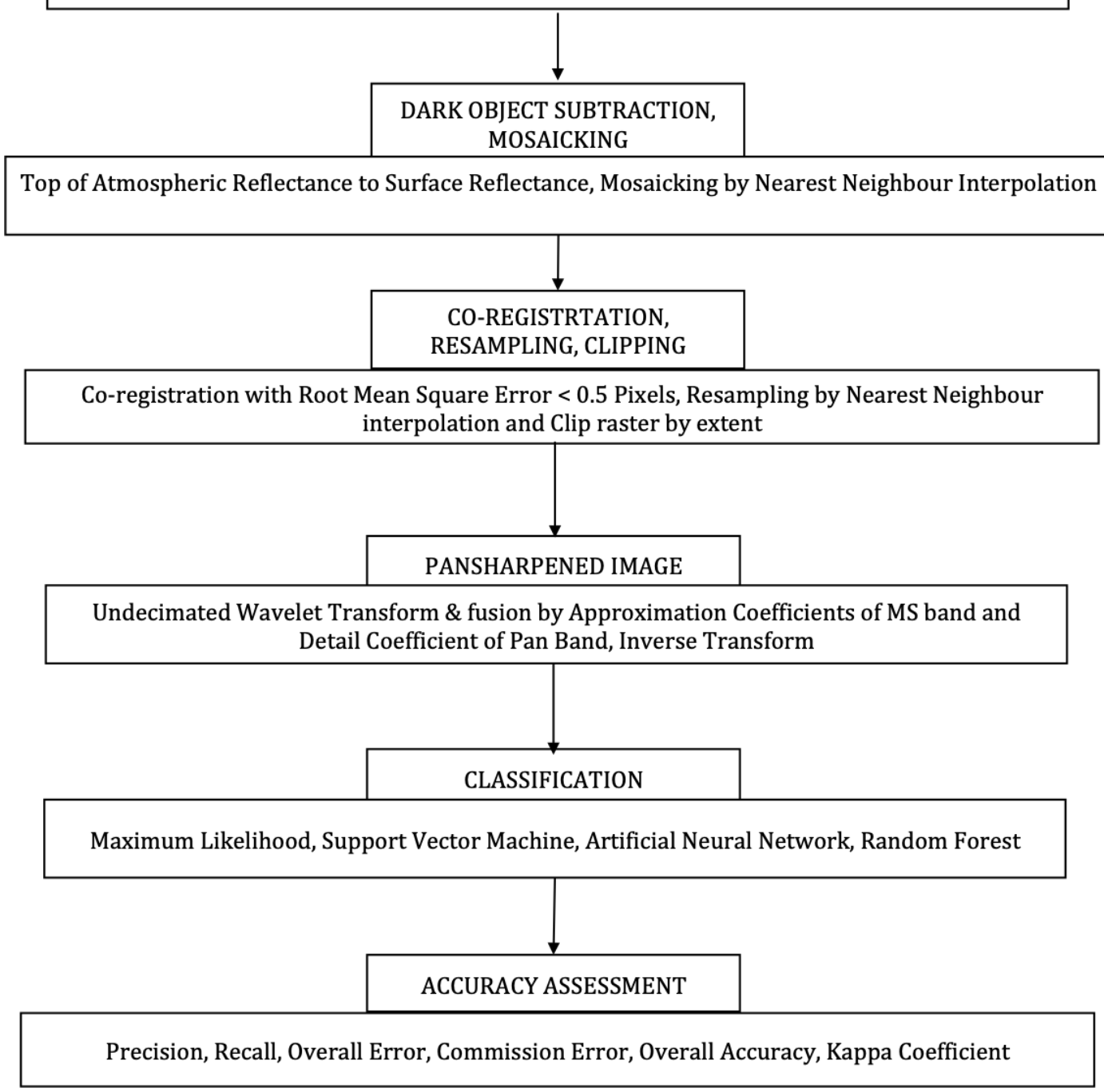

Fig. 2: Flow diagram of overall Methods used in this Study

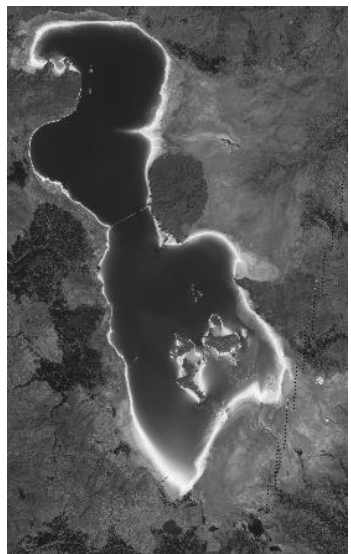

(a)

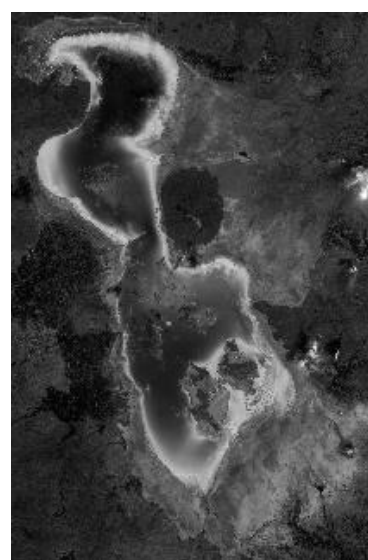

(b)

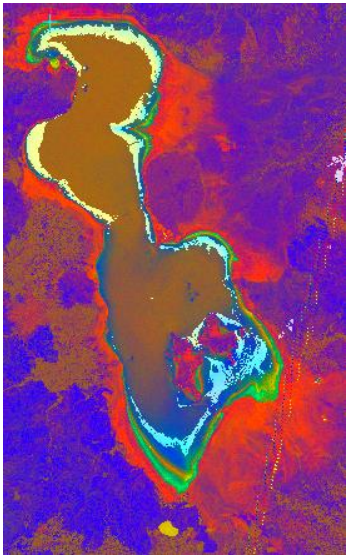

(c)

Fig. 3: (a) Multispectral band (b) Panchromatic band, (c) Pansharpened Image 


\section{Random Forest Classifier for Extracting Water bodies from Pansharpened Image to Detect Surface Water Changes}

Table- 3: Land Cover Classes and its description

\begin{tabular}{cc}
\hline Class & Description \\
\hline Land area & Surface area that was dried before 2010 \\
Changed area & Surface area that was changed as land in \\
Water area & 2018 from water in 2010 \\
\hline
\end{tabular}

The images are then mosaicked and co-registered with root mean squared error (RMSE) of less than 0.5 pixels. Then the nearest neighbor interpolation is used to resample the multispectral band to the same size of panchromatic band. To perform fusion, the undecimated wavelet transform is applied to the multispectral bands of Landsat 5 and the panchromatic band of Landsat 8. The derived approximation coefficients of multispectral image and detail coefficients of panchromatic image were used to obtain the fused coefficients. Applying inverse transformation to the fused coefficients then generates the pansharpened image. The multispectral band, panchromatic band and pansharpened image are shown in fig.3 a - c.

Region growing algorithm is used for creating a training area and the selection of pixels are based on the spectral similarity of adjacent pixels. The spectral signatures (spectral characteristics) for each Regions of Interest (ROI) were used as reference land cover classes for classification. The classification is based on the comparison of spectral characteristics of each pixel with reference class [7]. The classification algorithms such as Maximum Likelihood, Support Vector Machine, Artificial Neural Network and Random Forest were employed to the undecimated wavelet transform based fused image. Using these classification techniques, the pansharpened image was labeled as three classes namely land area, changed area and water area. Land area is the surface area that was dried before 2010, changed area refers the surface area that was changed as land in 2018 from water in 2010, and water area refers the lake surface area in 2018.

Maximum Likelihood (ML) classification uses Bayes theorem to calculate the probability distributions in the form of multivariate normal models for the classes to check whether a pixel belongs to the land cover class [8]. The ML uses both variance and covariance of the class spectral signatures in assigning each pixel to any one of the classes. Maximum Likelihood is the most commonly used parametric classifier in multispectral images. Number of researchers have done an extensive study on this classification and published literature.

Support Vector Machine (SVM) classifier is a supervised classification approach, which can handle large raster images (multispectral image)[9]. The classification is done pixel by pixel based on the trained feature file. In this approach, a multiclass pair-wise classification strategy was performed. It involves input and an output layer, selecting a proper kernel is challenging and the choice of selection is based on the feature parameters. The default gamma value 0.143 is set in kernel function to control the error. Each input image is processed at its full resolution by assigning the value 0 to the pyramid function. All the pixels in an image were classified in to any of the class label by propoerly setting a classification probability threshold.

Artificial Neural Network (ANN) doesn't require any assumption on feature distribution and minimum or no a priori knowledge on the characteristics of land cover class [6]. The standard propagation based non-linear feed forward model is employed for supervised learning. ANN works better even with small training dataset, and it involves input layer, output layer and one or more hidden layer.

Random Forest, an ensemble learning based decision tree classifier seems to be effective in handling large volume of data with high accuracy and computational performance [10] [11]. A random sub-sample is considered and a classification procedure is done based on that sub-sample. This process is done for 10 iterations and the average is computed to enhance the accuracy and to avoid over-fitting. This approach provides better estimates and suits well for larger datasets.

\section{RESULTS AND DISCUSSION}

To assess the performance of the classification techniques, the metrics such as Overall Error, Commission Error, Precision, Recall, Overall Accuracy and Kappa Coefficient were computed [12]. The ANN, SVM and Random Forest techniques successfully separated the water, land and changed pixels compared to maximum likelihood. The results in table 4, clearly shows that the application of random forest classifier on pansharpened image obtained the high accuracy (fig. 4). The proposed approach integrating the undecimated wavelets transform based fusion and random forest classifier has the advantage of generating high resolution of $15 \mathrm{~m}$ from $30 \mathrm{~m}$-resolution multispectral band \& $15 \mathrm{~m}$-resolution panchromatic band, and at the same time highlights the changes with maximum accuracy. The performance evaluation of various assessment metrics is shown in fig. 5-8. 


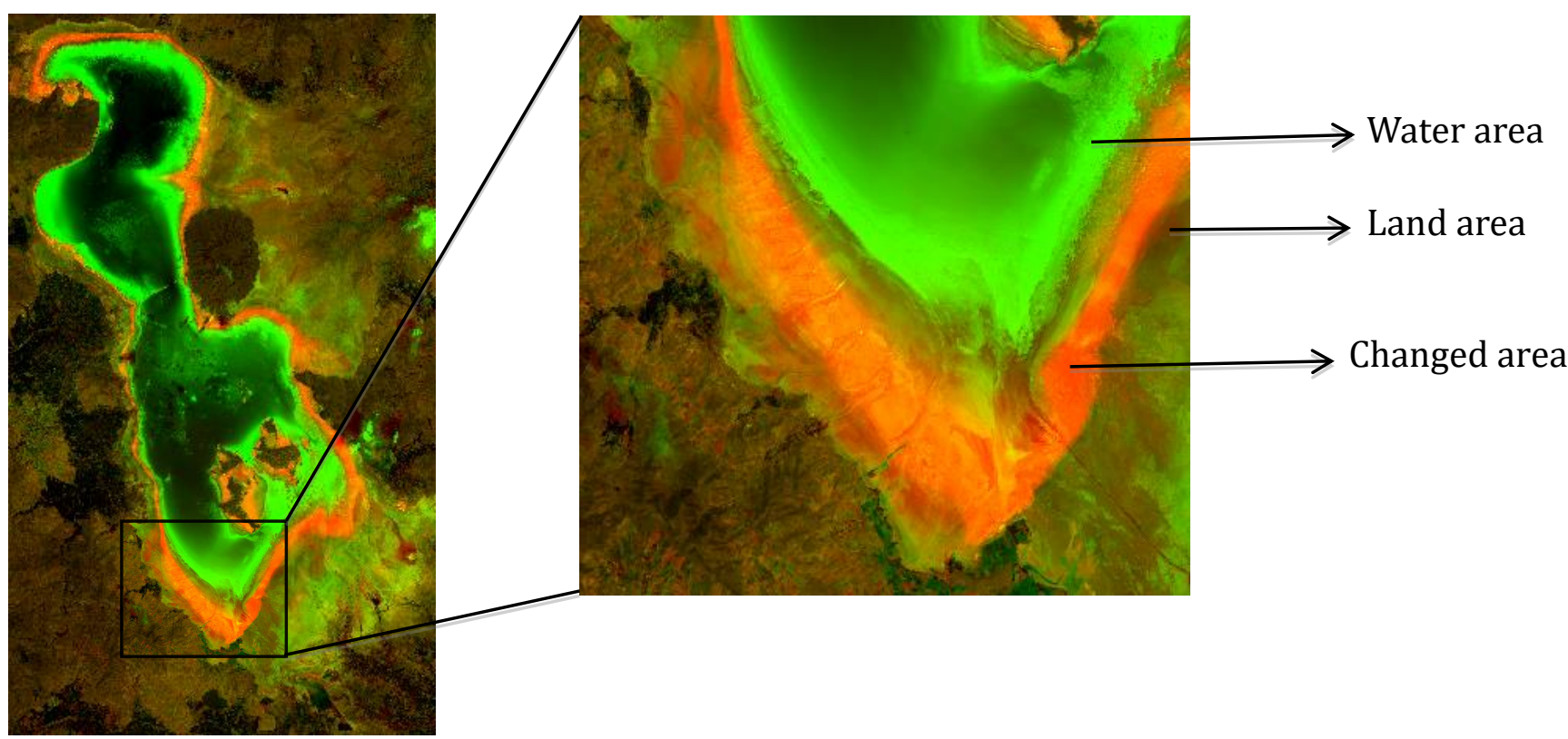

Fig. 4: Lake Surface area generated using Random Forest Classifier on Pansharpened Image

Table- 4: Results of Performance Metrics

\begin{tabular}{ccccccc}
\hline Method & $\begin{array}{c}\text { Overall } \\
\text { Error }\end{array}$ & $\begin{array}{c}\text { Commission } \\
\text { Error }\end{array}$ & Precision & Recall & $\begin{array}{c}\text { Overall } \\
\text { Accuracy }\end{array}$ & $\begin{array}{c}\text { Kappa } \\
\text { Coefficient }\end{array}$ \\
\hline ML & 1.57 & 1.91 & 0.04 & 0.71 & 98.15 & 0.87 \\
\hline SVM & 0.44 & 0.54 & 0.09 & 0.88 & 99.13 & 0.90 \\
\hline ANN & 0.34 & 0.53 & 0.09 & 0.89 & 99.37 & 0.91 \\
\hline $\begin{array}{l}\text { Random } \\
\text { Forest }\end{array}$ & 0.07 & 0.23 & 0.12 & 0.91 & 99.87 & 0.93 \\
\hline
\end{tabular}

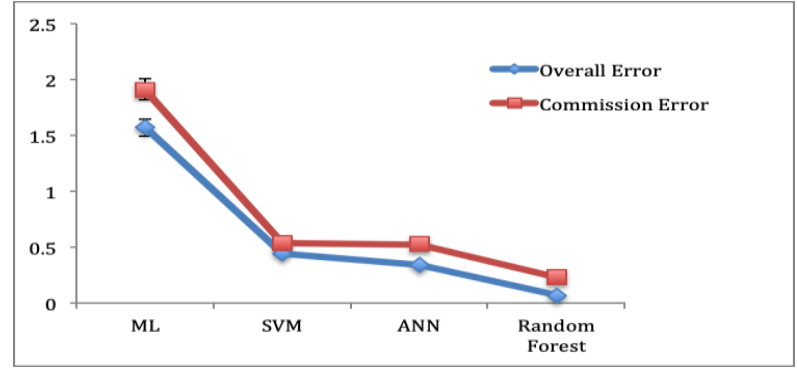

Fig. 5: Performance evaluation of overall error and commission error

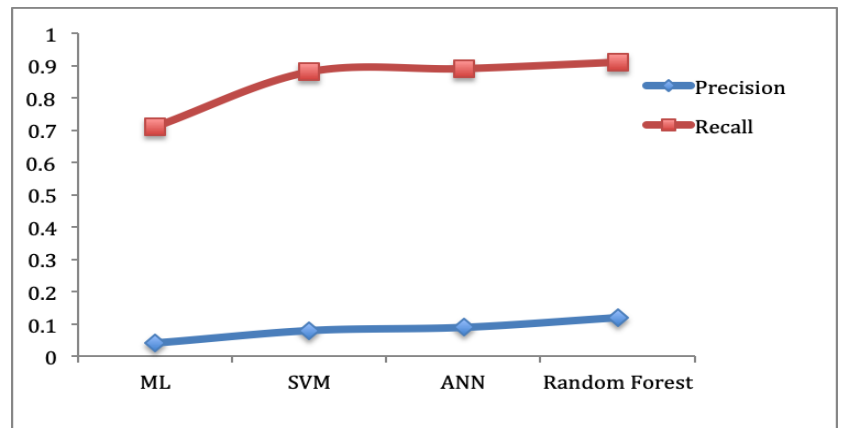

Fig. 6.: Performance evaluation of precision and recall

Retrieval Number: A2039109119/2019@BEIESP

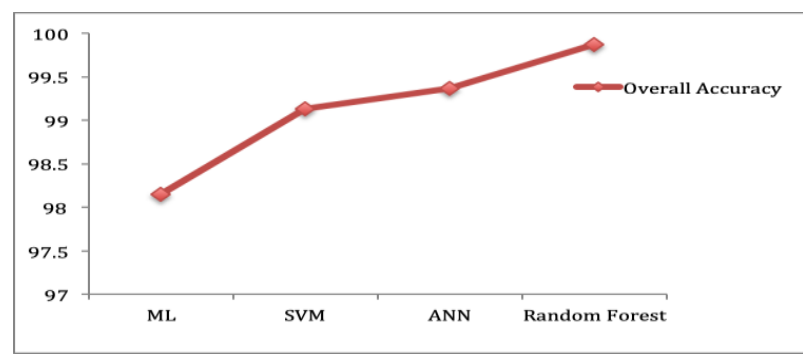

Fig. 7: Performance evaluation of overall accuracy

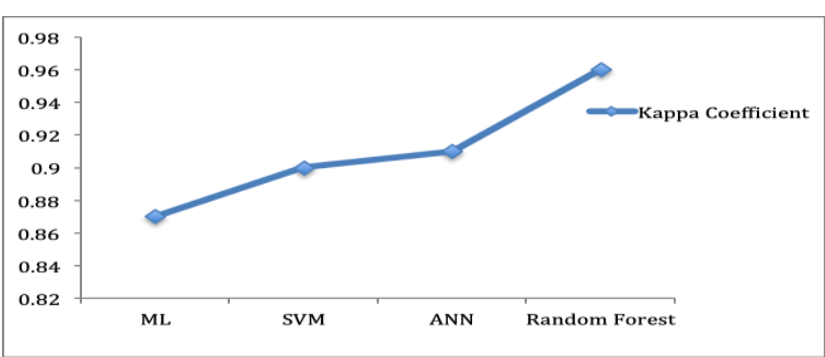

Fig. 8: Performance evaluation of kappa coefficient

Published By:

Blue Eyes Intelligence Engineering

\& Sciences Publication

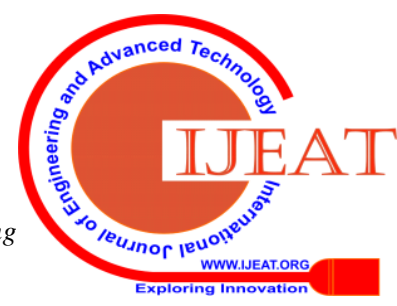




\section{Random Forest Classifier for Extracting Water bodies from Pansharpened Image to Detect Surface Water Changes}

\section{CONCLUSION}

The methodology for surface water extraction and change detection integrating the undecimated wavelet transform based fusion and random forest classifier is designed. The proposed methodology is applied on multi-temporal multi-spectral landsat 8 and landsat 5 images of Lake Urmia in North west of Iran. The pansharpened image highlights the changes in lake surface area between the years 2010 and 2018. Thus the potential of random forest technique has been explored in classifying and analyzing the multispectral images. The efficiency of the proposed approach is proved by comparing with other classification techniques such as maximum likelihood, support vector machine and artificial neural network, based on spectral signatures of various classes like land area, changed area and water area. In conclusion, the proposed methodology has proved to be efficient in extracting the water pixels and detecting the changes in multispectral Landsat images.

\section{REFERENCES}

1. H. Zhou, H. Jiang, and Q. Huang, "Landscape and water quality change detection in urban wetland: A post-classification comparison method with IKONOS data," in Procedia Environmental Sciences, 2011, vol. 10, no. PART B, pp. 1726-1731.

2. H. B. Mitchell, Image fusion: Theories, Techniques and Applications. Springer, 2010

3. S. Li, X. Kang, L. Fang, J. Hu, and H. Yin, "Pixel-level image fusion: A survey of the state of the art," Information Fusion, 2017.

4. V. Yilmaz and O. Gungor, "Fusion of very high-resolution UAV images with criteria-based image fusion algorithm," Arabian Journal of Geosciences, 2016.

5. K. Rokni, A. Ahmad, A. Selamat, and S. Hazini, "Water Feature Extraction and Change Detection Using Multitemporal Landsat Imagery," pp. 4173-4189, 2014.

6. K. Rokni, A. Ahmad, K. Solaimani, and S. Hazini, “A new approach for surface water change detection: Integration of pixel level image fusion and image classification techniques," International Journal of Applied Earth Observations and Geoinformation, vol. 34, pp. 226-234, 2015.

7. D. D. Nguyen, "Water Body Extraction From Multi Spectral Image By Spectral Pattern Analysis," ISPRS - International Archives of the Photogrammetry, Remote Sensing and Spatial Information Sciences, vol. XXXIX-B8, no. September, pp. 181-186, 2012.

8. A. Ahmad and S. Quegan, "Analysis of maximum likelihood classification on multispectral data," Applied Mathematical Sciences, vol. 6, no. 129-132, pp. 6425-6436, 2012.

9. D. Wang, B. Wan, P. Qiu, Y. Su, Q. Guo, and X. Wu, “Artificial mangrove species mapping using Pléiades-1: An evaluation of pixel-based and object-based classifications with selected machine learning algorithms," Remote Sensing, vol. 10, no. 2, 2018.

10. Z. Shao, Y. Zhang, L. Zhang, Y. Song, and M. Peng, "Combining spectral and texture features using random forest algorithm: Extracting impervious surface area in Wuhan," International Archives of the Photogrammetry, Remote Sensing and Spatial Information Sciences ISPRS Archives, vol. 41, no. July, pp. 351-358, 2016.

11. E. Scornet, "A Random Forest Guided Tour," no. March, 2016.

12. A. M. Lal and S. Margret Anouncia, "Semi-supervised change detection approach combining sparse fusion and constrained k means for multi-temporal remote sensing images," Egyptian Journal of Remote Sensing and Space Science, 2015. 